\title{
Case Report and Literature Review
}

\section{Total Body Irradiation as Conditioning Regimen for Haematopoietic Progenitor Cell Transplant: A Case Report and Literature Review}

\author{
Alonso Sánchez Diana" and Lloret Sáez-Bravo Marta \\ Radiation Oncology Department, Hospital Universitario de Gran Canaria Doctor Negrin, Gran Canaria, Spain

\section{A RTICLE INFO} \\ Article history: \\ Received: 22 July, 2020 \\ Accepted: 1 August, 2020 \\ Published: 12 August, 2020 \\ Keywords: \\ Total body irradiation \\ progenitor cell transplant \\ volumetric-radiotherapy \\ modulated intensity radiotherapy \\ leukaemia

\begin{abstract}
A B S T R A C T
This case study presents a young adult man with lymphoblastic leukaemia B who required total body irradiation (TBI) as a conditioning regimen for haematopoietic progenitor cell transplant (HPT) as the only curative treatment option for his oncological disease. TBI was carried out with personalised patient immobilisation, three-dimensional simulation, radiophysical planning and dosimetric calculations. A total of $12 \mathrm{~Gy}$ were prescribed to be administered twice a day for 3 days in a row by means of volumetric modulated intensity radiotherapy with several isocentres. The best technique for the administration of this radiotherapy is discussed because, although the clinical efficacy of the administration of this body dose has the technique used in this case (multi-isocentre volumetric-radiotherapy), which allows a better doses distribution, reducing the dose in organs at risk (OARs) and producing less toxicity, and therefore allowing the inclusion of patients who would not tolerate a conventional TBI. In addition, this method would allow the dose to be scaled up locally in higher areas at risk with better results in the disease control.
\end{abstract} \\ been well known for decades, technological advances have brought us new possibilities when compared to \\ the traditional TBI (bilateral with horizontal photon beam and absorbent screen). These advances include
}

\section{Introduction}

TBI consists of administering ionising radiation to the entire body for its conditioning for HPT [1]. It aims to ensure the immunosuppression needed to prevent rejection of haematopoietic progenitors or "graftversus-host disease" (immunoablative function), create space in the bone marrow (myeloablative) and eradicate tumour clonogens (cytotoxic). Due to the high radio sensitivity of both the haematopoietic and the tumour tissue, an effective dose can be achieved without damaging the rest of the body's tissues and therefore with acceptable and controllable toxicity.

Haematopoietic and tumour tissue has a high alpha/beta, much higher than most of the organs and tissues in the rest of the body which we would consider to be OAR [2]. Therefore, in addition to requiring relatively low doses to those used in radiotherapeutic oncology, we could achieve this dose in an optimal way with accelerated hypofractionated fractions (which would increase lethal injuries in the target tissues and allow the repair of sublethal injuries in OAR. Despite the knowledge of this branch of radiobiology and the proven effectiveness of TBI over decades of experience, there is still no consensus on the most appropriate fractionation. Likewise, in recent times new radiotherapy techniques have been developed, increasing the possibilities of how to administer the dose, but no agreement has been reached regarding the technique to be used.

\section{Case Report}

This is a 22-year-old patient with no medical or surgical history of interest who was transferred from the Haematology Department for a TBI for Acute Lymphoblastic Leukaemia B with exclusive involvement of the central nervous system (CNS) and who requires allogenic HPT. It has the translocation (9;22) (q34.1;q11.2); BCR-ABL1 with CNS and

*Correspondence to: Alonso Sánchez Diana, Radiation Oncology Department, Hospital Universitario de Gran Canaria Doctor Negrin, Calle Plaza Barranco de la Ballena S/N 35010, Gran Canaria, Spain; Email: dianaalonsosanchez@hotmail.com 
bone marrow involvement (at molecular level). He received prior and induction chemotherapy according to PETHEMA LAL Ph+ protocol for patients under 50 years old. Two lumbar punctures were performed weekly until confirmation of the disinfiltration of the cerebrospinal fluid by immunofluorescence. After TBI, consolidation chemotherapy was administered according to the same protocol, both intravenously and intrathecally. The transplant performed was of haploidy HPT from the patient's mother.

With regard to the TBI, the patient showed an excellent general condition after a thorough physical examination which was completely normal when assessed by Radiation Oncology (RON). Consequently, upon the informed consent, the simulation of the volumetric modulated arc TBI was carried. The first step was to place the patient in the position in which the simulation CT was to be performed and each radiotherapy session in the RON simulation CT room

This is the first step in treatment planning, in which the patient's positioning will be set out for the correct performance of the treatment, optimising dosimetry and minimising the differences in intra and interfractional positioning of the radiotherapy. We proceed to the implementation and assembly of immobilisation systems with the patient in a supine position with the arms close to the body and slightly bent and the palms of the hands oriented centrally to minimise the air spaces between the arms and the trunk (Figure 1). The appropriate headrest is also carefully chosen to keep the neck in a neutral position. A long thermoplastic mask and an indexed vacuum mattress are manufactured, and the legs and feet are held in place with a customised elastic tubular bandage for closeness, immobilisation and ease of reproduction.

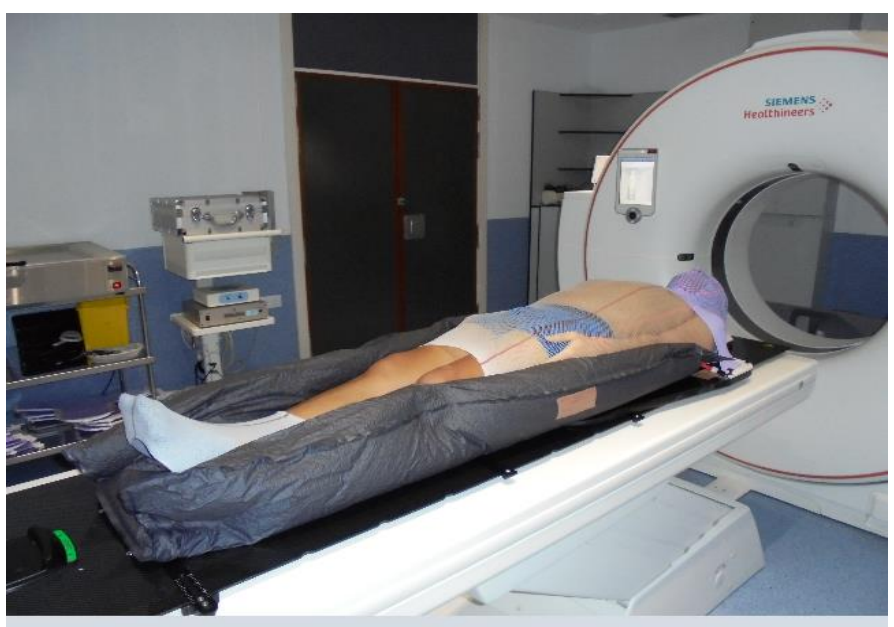

Figure 1: Patient's positioning with immobilisation systemsin CT roomcan be observed in this photograph above.

Subsequently, the baseline tattoos were performed ( 3 on the chest and 3 on the pelvis). Finally, all their characteristics were written down in detail and photos were taken of the patient's positioning and face, which will be entered into the linear accelerator software. With the personalised immobilisers manufactured, the simulation CT was carried out in the CT of the PET-CT of the Nuclear Medicine Department to obtain axial images of the whole body. On these axial images and their coronal, sagittal and three-dimensional reconstruction, the contouring of the organs at risk and the volume planning, dosimetry and planning were carried out by the Radiophysical Unit with the Eclipse ${ }^{\mathrm{TM}}$ planning system.

The OARs which were contoured were the lungs (separately and building a structure called the lungs using the addition of both, and building another structure which will be the addition of both minus an internal margin of $0.5 \mathrm{~cm}$ ), the liver, the kidneys (separately) and the lens (separately). The planning treatment volume (PTV) was obtained by subtracting from the patient's contour with an internal margin of $0.5 \mathrm{~cm}$ the structure formed by subtracting an internal margin of $0.5 \mathrm{~cm}$ from the addition of the lungs. For this PTV, 12 Gy/3 days in a row were prescribed ( 2 sessions/day separated by a minimum of $6 \mathrm{~h}$ ). Dosimetric restrictions in OARs were indicated for lungs- 0.5 (dose between 8 - 10 Gy, maximum dose $15 \mathrm{~Gy}$, dose in $1500 \mathrm{cc}$ less than $13.5 \mathrm{~Gy}$ ), liver (maximum dose $24 \mathrm{~Gy}$, dose in $700 \mathrm{cc}$ less than $21 \mathrm{~Gy}$ ), kidneys (dose in $200 \mathrm{cc}$ less than $19 \mathrm{~Gy}$ ) and lens (maximum dose 9 Gy).
After a joint assessment by the physicist and the radiation oncologist, the treatment plan was approved. This plan consisted of 3 isocentres in the upper half of the body and 3 isocentres in the lower half of the body with several high-energy photon arcs (6 MV) in each one of them. Thus, dosimetry data were: average lungs- 0.5 dose $=9.78 \mathrm{~Gy}$, maximum lungs- 0.5 dose $=13.15 \mathrm{~Gy}$, V60 lungs- $0.5=9.49 \mathrm{~Gy}$, D1500cc lungs$0.5=9.82 \mathrm{~Gy}$, maximum liver dose $=13.6 \mathrm{~Gy}$, dose 200c kidneys $=0$ Gy in both, maximum dose in lens $=6.3 \mathrm{~Gy}$, average dose for thyroid = 14.24 Gy, maximum dose for upper half-body $=14.44 \mathrm{~Gy}$ and maximum dose for lower half-body $=14.39 \mathrm{~Gy}$. All this with appropriate PTV coverage.

The treatment was conducted in a CLINAC DHX linear accelerator with Varian ${ }^{\circledR}-120$ multilayers after an image control for each isocentre prior to each part of the radiotherapy session using the on-board imager (OBI) system. Each treatment session had two parts since, first, the upper part of the patient was treated and then the lower part (with feet towards the gantry) due to the limited mobility in the longitudinal direction of the treatment table. During said treatment, the patient remained admitted and received supportive medication to monitor for infection or toxicity. Prophylactically, $8 \mathrm{mg}$ of intravenous ganisentron was administered half an hour before each treatment session. There was no toxicity of grade 2 nor 3 according to the radio-induced RTOG score, neither acute nor in the medium term. 


\section{Discussion}

As in all treatments with ionising radiation, the aim is to radiate the target tissue up to an effective dose while safeguarding as much as possible the dose administered to the OARs in order to avoid both short-term and long-term toxicities. TBI can be administered before or after chemotherapy but always sequentially to decrease the potential toxicity of both. Different techniques can be used. One of them, the oldest, is achieved by means of 2 fixed radiation beams, with customised lung protections using Cerrobend ${ }^{\circledR}$ (lead alloy) moulds with the shape of both lung fields customised thanks to simple X-rays, absorbent screen and in vivo dosimetry. Another newer technique would be through simulation and three-dimensional planning and modulated intensity whose inverse dosimetry allows us to restrict the dose in the OARs such as the lungs and to calculate this without the need for in vivo dosimetry and prior to radiating the patient [3].

In the TBI, the target organs are the haematopoietic progenitors and the leukemic tumour tissue located mainly in the bones but also distributed throughout the rest of the body in its vascularisation. The OARs would be, theoretically, all the others. Special emphasis needs to be placed on the long-term toxicities when dealing with patients at an early age, usually young adults, and having this treatment a curative purpose. The most important organs at risk would be the lungs, liver, kidneys and lens because of the possibility of producing long-term side effects with doses in the ranges we would apply in these treatments. Actually, the lens are taken into account because of their great radio sensitivity but in no case would they be dose limiters since the cataracts that might be caused are easily correctable with the performance of a bilateral phakectomy.

The main organ which has always been recommended to be protected are the lungs in order to avoid pulmonary fibrosis as a long-term toxicity. However, the lung should not have the lowest possible dose but a minimum due to its great vascularisation and for this reason it is considered appropriate to have a dose in the lung volume of 8-10 Gy and this is what is applied in most Spanish centres. The use of a bolus for the arrival of higher doses to skin tissue is controversial, although the absorbent screen is traditionally used for this purpose. The aim of this skin radiation would be the lymphocytes found in the dermis, even though this would lead to greater skin toxicity such as radiodermatitis in the short term and skin fibrosis in the long term. From the beginning of the technique, the single-dose (10 Gy) treatment regimen was quickly discarded because of the incidence of pulmonary fibrosis [4]. Accelerated hyperfractionation was used, which demonstrated less toxicity without compromising anti-tumour effectiveness or even increasing it by inducing partial synchronisation of the leukemic tissue cell cycle [5]. The radiobiological characteristics of lung tissue (2.8 Gy alpha/beta) allow it to rapidly repair sublethal damage (with doses spaced at 4-6 h apart) unlike the alpha/beta of tumour cells or bone marrow. Thus, the treatment is optimised by administering a lower dose per fraction and achieving a higher total dose. [6-9].

Acute toxicity is characteristic of tissues with a high alpha-beta index, with a high rate of cell replication. We will thus find alterations in the digestive mucosa (nausea and vomiting) and in glandular tissues (xerostomia). At an early stage, they may also present febrile syndrome due to pyrogens released by both healthy and tumour cells, renal failure, arrhythmias, cardiomyopathy, haemorrhagic cystitis, interstitial pneumonitis, alveolar haemorrhage, veno-occlusive disease, infections, alopecia, altered nail growth and mumps. In the long term, endocrine and gonadal failures (growth and fertility alterations in children and hypothyroidism in adults), pulmonary alterations (from mild pneumonitis to fibrosis), cardiomyopathy, arrhythmias, hepatic sinusoidal obstruction syndrome, cataracts, neurocognitive and learning alterations and second tumours may appear [10].

The incidence of these complications responds to a large number of factors, such as the fractionation and the dose of the radiotherapy treatment, but also to the dose and type of conditioning with chemotherapy used, the concomitant use of other drugs such as steroids, the compatibility of the donor with the host, the existence of infections during treatment, the patient's underlying pathology, his or her comorbidities and toxic habits. There is currently no national agreement as to the technique for undertaking this treatment which, although it is not the most frequent in Radiation Oncology Departments, is an effective and safe therapeutic method that we should continue to use and implement.

\section{Conflicts of Interest}

None.

\section{Funding}

None.

\section{REFERENCES}

1. N P Mendenhall, W D Noyes, R R Million (1989) Total body irradiation for stage II-IV non-Hodgkin's lymphoma: ten-year followup. J Clin Oncol 7: 67-74. [Crossref]

2. J M Cosset, G Socie, B Dubray, T Girinsky, A Fourquet et al. (1994) Single dose versus fractionated total body irradiation before bone marrow transplantation: radiobiological and clinical considerations. Int J Radiat Oncol Biol Phys 30: 477-492. [Crossref]

3. Springer A, Hammer J, Winkler E, Track C, Huppert R et al. (2016) Allogenic marrow transplantation in patients with chronic myeloid leukemia. Radiat Oncol 11: 46

4. R A Clift, C D Buckner, F R Appelbaum, E Bryant, S I Bearman et al. (1991) Allogenic marrow transplantation in patients with chronic myeloid leukemiain the chronic phase: a randomized trial of two irradiation regimens. Blood 77: 1660-1665. [Crossref]

5. P Latini, C Aristei, F Aversa, F Checcaglini, E Maranzano et al. (1991) Lung damage following bone marrow transplantation after hyperfractionated total body irradiation. Radiother Oncol 22: 127-132. [Crossref]

6. Barrett A (1982) Total body irradiation before bone marrow transplantation: a review. Clin Radiol 33: 131-135. [Crossref]

7. J M Cosset, T Girinsky, E Malaise, M P Chaillet, J Dutreix (1990) Clinical basis for TBI fractionation. Radiother Oncol 18: 60-67. [Crossref]

8. Sagus Sampath, Timothy E Schultheiss, Jeffrey Wong (2005) Dose response and factors related to interstitial pneumonitis after bone 
marrow transplant. Int J Radiat Oncol Biol Phys 63: 876-884. [Crossref]

9. S V Harden, D S Routsis, A R Geater, S J Thomas, C Coles et al. (2001) Total body irradiation using a modified standing technique: a single institution 7-year experience. Br J Radiol 74: 1041-1047. [Crossref]
10. R Yan, L J Peters, E L Travis (1991) Cyclophosphamide 24 hours before or after total body irradiation: effects on lung and bone marrow. Radiother Oncol 21: 149-156. [Crossref] 